Anaesthesist 2012 - 61:95-96

DOI 10.1007/s00101-012-1995-3

Online publiziert: 23. Februar 2012

(c) Springer-Verlag 2012

\section{Byhahn ${ }^{1} \cdot$ W. Ummenhofer ${ }^{2}$}

${ }^{1}$ Klinik für Anästhesiologie, Intensivmedizin und Schmerztherapie,

Klinikum der J.W. Goethe-Universität, Frankfurt

${ }^{2}$ Departement Anästhesie und Operative Intensivmedizin, Universitätsspital Basel

\title{
Ubi carbonii dioxidum, ibi vita est
}

In dieser Ausgabe von Der Anaesthesist gehen Timmermann et al. in einer Übersichtsarbeit auf die Wertigkeit der Kapnometrie und Kapnographie in der Notfallmedizin ein.

Hand aufs Herz: Brauchen wir diese technischen Spielereien aus dem OP denn jetzt auch noch in der präklinischen Situation? Intubieren können wir doch; das haben wir jahrelang schließlich auch ohne Kapnometrie bewiesen. Wozu gibt es schließlich in Deutschland ein Rettungssystem, das notarztbasiert arbeitet?

Schauen wir einmal über den Teich, wo die präklinische Notfallversorgung zwar "nur" durch Paramedics sichergestellt, aber zumindest regelmäßig durch selbstkritische Studien begleitet wird: Es wurden $12 \%$ [1] bzw. 17\% [2] unbemerkte ösophageale Fehlintubationen in Florida festgestellt, und auch in anderen Teilen der USA sieht es nicht viel besser aus. Im vergangenen Jahr publizierten Wang et al. [3] eine Arbeit, in der in 16 repräsentativen US-Bundesstaaten insgesamt knapp 4,4 Mio. Einsätze des Rettungsdienstes ausgewertet wurden. Von 8418 Intubationsversuchen waren wiederum lediglich $77 \%$ erfolgreich. Auch die als problemlos eingeschätzte Verwendung extraglottischer Atemwegshilfen verzeichnete nur eine Erfolgsrate von $87 \%$ ! Sie werden uns sicherlich zustimmen, dass dies besorgniserregende Zahlen sind - aber sie stammen ja aus einer vermeintlich anderen Welt.

Nach einigen Fallberichten unbemerkter ösophagealer Fehlintubationen durch Notärzte in Österreich [4] haben im Jahr 2007 Timmermann et al. [5] auch einmal dem hochgelobten deutschen Notarztsystem den Spiegel vorgehalten und die mut- maßlich korrekte Tubuslage bei Patienten, die bereits von einem bodengebundenen Notarzt intubiert worden waren, durch den nachgerückten Hubschraubernotarzt verifizieren lassen. Es waren 7\% der Patienten unbemerkt ösophageal intubiert worden. Ob es für oder gegen das deutsche Notarztsystem spricht, dass die Rate unbemerkter ösophagealer Fehlintubationen von 17 bzw. 12\% in ParamedicSystemen auf immerhin $7 \%$ gesenkt werden konnte, möge jeder für sich selbst entscheiden.

\section{$\mathrm{CO}_{2}$-Messung die Lösung?}

Was hat das aber nun alles mit präklinischer $\mathrm{CO}_{2}$-Messung zu tun? Silvestri et al. [6] konnten beispielsweise zeigen, dass die Rate unbemerkter ösophagealer Fehlintubationen durch Paramedics auf null gesenkt werden konnte, wenn protokollgemäß eine kontinuierliche endtidale $\mathrm{CO}_{2}$ Messung verwendet wurde, und auch Timmermann et al. [5] forderten die obligate präklinische Tubuslagekontrolle mithilfe der Kapnometrie.

Um die Komplikationsraten im Atemwegsmanagement in allen 309 Kliniken des National Health Service (NHS) in Großbritannien u. a. auch auf der Intensivstation und in der Notaufnahme prospektiv zu erfassen, erfolgte in den Jahren 2008 und 2009 die Durchführung des Projekts Fourth National Audit Project of the Royal College of Anaesthetists and the Difficult Airway Society (NAP4). Insgesamt 51 kritische Zwischenfälle wurden auf Intensivstationen oder in Notaufnahmeeinheiten registriert; hierbei hatten $61 \%$ der Zwischenfälle auf der Inten- sivstation und $31 \%$ derer in den Notaufnahmen den Tod des Patienten oder einen hypoxischen Hirnschaden zur Folge. Auf der Intensivstation waren Probleme während der Tracheotomie oder bei tracheotomierten Patienten sowie unbemerkte ösophageale Fehlintubationen als Ursache führend, während in der Notaufnahme Intubationsschwierigkeiten und Aspiration den Großteil der Probleme begründeten. Die Autoren wiesen nach, dass bei $74 \%$ aller Zwischenfälle, die einen tödlichen Ausgang genommen oder einen permanenten Hirnschaden zur Folge gehabt hatten, keine Kapnometrie zur Anwendung gekommen war [7].

\section{Verweigern kann tödlich sein}

Auf allen seit 2007 neu zugelassenen deutschen Rettungsmitteln ist nunmehr eine exspiratorische $\mathrm{CO}_{2}$-Messung verbindlich vorgeschrieben.

Und noch einmal Hand aufs Herz: Obwohl Intubationsprobleme in der präklinischen Versorgung erwiesenermaßen wesentlich häufiger auftreten als unter kontrollierten Bedingungen im OP [8] - nutzen Sie die Kapnometrie oder Kapnographie, sofern Sie Ihnen zur Verfügung steht, präklinisch mit der gleichen Selbstverständlichkeit wie im OP? Oder anders herum: Würden Sie eine elektive Intubationsnarkose bei einem ASA-I-Patienten im OP beginnen und durchführen, wenn die exspiratorische $\mathrm{CO}_{2}$-Messung defekt wäre? Oder sogar die „rapid sequence induction" eines Traumapatienten im Schockraum? In allen Fällen lautet die ehrliche Antwort vermutlich "nein“. Dass diese leider noch immer weitver- 
breitete Ignoranz der präklinischen $\mathrm{CO}_{2}$ Messung nicht nur für den Patienten fatale Folgen haben, sondern im Schadensfall auch medikolegale Konsequenzen nach sich ziehen kann, muss jedem „Verweigerer" dieses Verfahrens klar sein.

Neben der Tubuslagekontrolle bietet die kontinuierliche $\mathrm{CO}_{2}$-Messung noch eine Reihe weiterer Möglichkeiten in der Versorgung von Notfallpatienten. Hier soll zunächst die indirekte hämodynamische Überwachung, insbesondere während des bodengebundenen Transports, genannt werden. Jeder Notarzt kennt das Problem während der Fahrt: Die Blutdruckmessung ist extrem störanfällig und liefert häufig keine validen Ergebnisse, die Plethysmographie ist bei kreislaufinstabilen oder zentralisierten Patienten ebenfalls nicht verwertbar, und der Abgleich des EKG-Bilds mit den zentralen Pulsen des Patienten ist oftmals auch nicht sicher möglich. Zumindest bei intubierten Patienten stellt die kontinuierliche $\mathrm{CO}_{2}$-Messung daher ein wertvolles, semiquantitatives Instrument zur HerzKreislauf-Überwachung dar. Weiterhin kann das Verfahren dazu beitragen, eine Hyper- oder Hypoventilation eines beatmeten Notfallpatienten zu erkennen und zeitnah zu korrigieren.

In der Klinik stellt die kontinuierliche $\mathrm{CO}_{2}$-Messung zur Überwachung der Respiration analgosedierter Patienten, z. B. in der Magnetresonanztomographie (MRT) oder bei anderen diagnostischen sowie interventionellen Eingriffen, ein Standardverfahren dar. Bekanntermaßen führt eine leichte Atemdepression durch eine Sedierung zunächst zu einer Hyperkapnie und erst sekundär zur Hypoxämie, die über die Pulsoxymetrie detektiert werden kann. Das Erkennen und die Korrektur einer iatrogen induzierten Hypo- und Hyperkapnie können gerade in der Notfallmedizin bei kardiopulmonal akut eingeschränkten Patienten von erheblicher Bedeutung sein. Im Gegensatz zu früher wird die Hyperventilation insbesondere auch beim Schädel-Hirn-Trauma sehr kritisch beurteilt [9].

\section{Nicht nur kennen, sondern auch anwenden!}

Sehr geehrte Leser, auch wenn die $\mathrm{CO}_{2}{ }^{-}$ Messung seit einigen Jahren auf Rettungsmitteln verpflichtend vorgehalten werden muss und gegenwärtig weitestgehend flächendeckend zur Verfügung steht, nutzt sie Ihren Patienten nur dann, wenn Sie sie auch anwenden. Was im klinischen Alltag seit vielen Jahren eine etablierte Selbstverständlichkeit ist, sollten und dürfen wir unseren Patienten im Notarztdienst und in der Notaufnahme nicht vorenthalten. Denken Sie einfach beim nächsten $\mathrm{Pa}$ tienten daran, auf den kleinen Knopf „et$\mathrm{CO}_{2}{ }^{\text {“ }} \mathrm{zu}$ drücken! Vielleicht ist es aber auch wichtig, dass nicht nur das Individuum an seine Sorgfältigkeitspflicht erinnert wird, denn, wenn die Euronorm 1789 das Vorhalten der Kapnographie auf Rettungsfahrzeugen verbindlich vorschreibt, dann sollten Sie als verantwortlicher ärztlicher Leiter auch sicherstellen, dass Ihr medizinisches Personal dieses Sicherheitsmonitoring bei beatmeten Patienten einsetzt. Die Balance zwischen Eigenverantwortung des Individuums und Verantwortung des organisierenden Systems muss in Richtung der organisatorischen Strukturen verschoben werden, die mehr als bisher dafür Sorge tragen müssen, dass sich auch in der Medizin Fehlerkultur zur Sicherheitskultur entwickeln kann.

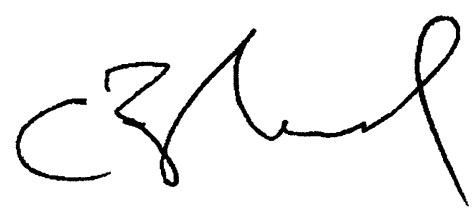

C. Byhahn

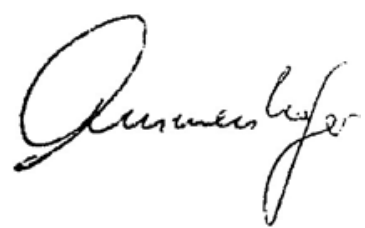

W. Ummenhofer

\section{Korrespondenzadresse}

Prof. Dr. C. Byhahn

Klinik für Anästhesiologie, Intensivmedizin und Schmerztherapie,

Klinikum der J.W. Goethe-Universität

Theodor-Stern-Kai 7, 60590 Frankfurt

c.byhahn@em.uni-frankfurt.de

Interessenkonflikt. Der korrespondierende Autor gibt an, dass kein Interessenkonflikt besteht.

\section{Literatur}

1. Katz SH, Falk JL (2001) Misplaced endotrachealtubes by paramedics in an urban emergency medical services system. Ann Emerg Med 37:32-37

2. Cobas MA, De la Peña MA, Manning R et al (2009) Prehospital intubations and mortality: a level 1 trauma center perspective. Anesth Analg 109:489493

3. Wang HE, Mann NC, Mears G et al (2011) Out-ofhospital airway management in the United States. Resuscitation 82:378-385

4. von Goedecke A, Keller C, Voelckel WG et al (2006) Maskenbeatmung als Rückzugsstrategie zur endotrachealen Intubation. Anaesthesist 55:70-79

5. Timmermann A, Russo SG, Eich C et al (2007) The out-of-hospital esophageal and endobronchial intubations performed by emergency physicians. Anesth Analg 104:619-623

6. Silvestri S, Ralls GA, Krauss B et al (2005) The effectiveness of out-of-hospital use of continuous end-tidal carbon dioxide monitoring on the rate of unrecognized misplaced intubation within a regional emergency medical services system. Ann Emerg Med 45:497-503

7. Cook TM, Woodall N, Harper C et al (2011) Major complications of airway management in the UK: results of the Fourth National Audit Project of the Royal College of Anaesthetists and the Difficult Airway Society. Part 2: Intensive care and emergency departments. Br J Anaesth 106:632-642

8. Russo SG, Zink W, Herff H, Wiese CH (2010) Tod durch (k)einen Atemweg. Anaesthesist 59:929939

9. Davis DP, Peay J, Sise MJ et al (2010) Prehospital airway and ventilation management: a trauma score and injury severity score-based analysis. J Trauma 69: 294-301 